\title{
Existence of Nonoscillatory Solutions of a Class of Nonlinear Dynamic Equations with a Forced Term
}

\author{
Shanliang Zhu, Xinli Zhang \\ College of Mathematics and Physics, Qingdao University of Science and Technology, Qingdao, China \\ Email: zhushanliang77@163.com
}

Received June 26, 2012; revised July 19, 2012; accepted July 30, 2012

\begin{abstract}
In this paper, we consider the following forced higher-order nonlinear neutral dynamic equation

$[x(t)+p(t) x(\tau(t))]^{\Delta^{m}}+f\left(t, x\left(\tau_{1}(t)\right), x\left(\tau_{2}(t)\right), \cdots, x\left(\tau_{k}(t)\right)\right)=q(t), t \in\left[t_{0}, \infty\right)_{\mathbb{T}}$

on time scales. By using Banach contraction principle, we obtain sufficient conditions for the existence of nonoscillatory solutions for general $p(t)$ and $q(t)$ which means that we allow oscillatory $p(t)$ and $q(t)$. We give some examples to illustrate the obtained results.
\end{abstract}

Keywords: Dynamic Equation; Higher Order; Non-Oscillation; Time Scale; Neutral

\section{Introduction}

The study of dynamic equations on time scales, which has recently received a lot of attention, was introduced by Stefan Hilger in his Ph.D. thesis in 1988 in order to unify continuous and discrete analysis [1]. Dynamic equations on time scales have an enormous potential for modelling a variety of applications such as in population dynamics. Several authors have expounded on various aspects of this new theory, see the survey paper by Agarwal, Bohner, O'Regan and Peterson [2] and references cited therein. A book on the subject of time scales, by Bohner and Peterson [3], summarizes and organizes much of the time scale calculus. We refer also to the last book by Bohner and Peterson [4] for advances in dynamic equations on time scales.

Recently, much attention is concerned with oscillation and nonoscillatory solutions for dynamic equations on time scales [5-12].

In Li and Zhang [6] studied the existence of nonoscillatory solutions to neutral dynamic equation

$$
\begin{aligned}
& {[x(t)+p(t) x(\tau(t))]^{\Delta^{n}}} \\
& +f_{1}\left(t, x\left(\tau_{1}(t)\right)\right)-f_{2}\left(t, x\left(\tau_{2}(t)\right)\right)=0 .
\end{aligned}
$$

Li, Han, Sun and Yang [10] established the existence of nonoscillatory solutions to the following second order neutral delay dynamic equation

$$
\begin{aligned}
& {\left[x(t)+p(t) x\left(\tau_{0}(t)\right)\right]^{\Delta \Delta}} \\
& +q_{1}(t) x\left(\tau_{1}(t)\right)-q_{2}(t) x\left(\tau_{2}(t)\right)=\mathrm{e}(t) .
\end{aligned}
$$

Zhang and Sun [13] studied the existence of nonoscillatory solutions of the forced nonlinear difference equation

$$
\Delta\left(x_{n}-p_{n} x_{\tau(n)}\right)+f\left(n, x_{\sigma(n)}\right)=q_{n} .
$$

Zhou and Zhang [14] obtained some sufficient conditions of nonoscillatory solutions for the higher order delay difference equation with positive and negative coefficients

$$
\Delta^{m}\left(x_{n}+c x_{n-k}\right)+p_{n} x_{n-r}-q_{n} x_{n-l}=0 .
$$

$\mathrm{Lu}$ [15] obtained some necessary and sufficient conditions for the existence of nonoscillatory solutions for the following first order neutral equation

$$
\left(x(t)-\sum_{i=1}^{m} p_{i}(t) x\left(h_{i}(t)\right)\right)^{\prime}+\sum_{j=1}^{n} f_{j}\left(t, x\left(g_{\mathrm{j}}(t)\right)\right)=Q(t) \text {. }
$$

Motivated by these works, in this paper, we consider the higher-order nonlinear neutral dynamic equation

$$
\begin{aligned}
& {[x(t)+p(t) x(\tau(t))]^{\lambda^{m}}} \\
& +f\left(t, x\left(\tau_{1}(t)\right), x\left(\tau_{2}(t)\right), \cdots, x\left(\tau_{k}(t)\right)\right)=q(t),
\end{aligned}
$$

where $t \in\left[t_{0}, \infty\right)_{\mathbb{T}}, \quad m \in \mathbb{N}, \sup \mathbb{T}=\infty$. We assume $p, q \in C_{r d}\left(\left[t_{0}, \infty\right)_{\mathbb{T}}, \mathbb{R}\right)$ and allow $p(t)$ and $q(t)$ to be oscillatory. $\tau, \tau_{i} \in C_{r d}\left(\left[t_{0}, \infty\right)_{\mathbb{T}}, \mathbb{T}\right)$ satisfy $\lim _{t \rightarrow \infty} \tau(t)=\lim _{t \rightarrow \infty} \tau_{i}(t)=+\infty, i=1,2, \cdots, k,$. $f\left(t, u_{1}, u_{2}, \cdots u_{k}\right) \in C\left(\mathbb{T} \times \mathbb{R}^{k}, \mathbb{R}\right)$ is nondecreasing for 
$u_{j}$ and $u_{1} f\left(t, u_{1}, u_{2}, \cdots u_{k}\right) \geq 0$ for

$$
u_{1} u_{j} \geq 0, j=1,2, \cdots, k \text {. }
$$

We recall $x$ is a solution of Equation (1) provided that $x(t)+p(t) x(\tau(t))$ is $m$ times differentiable, and $x$ satisfies Equation (1), A solution $x$ of Equation (1) is called nonoscillatory if $x$ is of one sign when eventually.

\section{Existence Results for Nonoscillatory Solutions}

In this section, we establish sufficient conditions of the existence of nonoscillatory solutions for Equation (1). First we define a sequence of functions $g_{k}(s, t), k \in \mathbb{N}_{0}$ as follows:

$$
g_{0}(s, t) \equiv 1, g_{k+1}(s, t)=\int_{t}^{s} g_{k}(\sigma(\tau), t) \Delta \tau .
$$

For $g_{k}(s, t)$, we have the following Lemma.

Lemma 2.1. (Li and Zhang [6]) Assume $s$ is fixed, and let $g_{k}^{\Delta}(s, t)$ be the derivative $g_{k}(s, t)$ with respect to $t$. Then

$$
g_{k}^{\Delta}(s, t)=-g_{k-1}(s, t), k \in \mathbb{N}, t \in \mathbb{T}^{k} .
$$

Let $B C$ denote the Banach space of all bounded functions $x(t), t \geq t_{0}$, with the norm $\|x\|=\sup _{t \geq t_{0}},|x(t)|<\infty$. We will use the following assumptions:

(i) there exists $\alpha>0$ such that

$$
\begin{aligned}
& \left|f\left(t, u_{1}, u_{2}, \cdots u_{k}\right)-f\left(t, v_{1}, v_{2}, \cdots v_{k}\right)\right| \\
& \leq L(t) \max _{1 \leq i \leq k}\left|u_{i}-v_{i}\right|
\end{aligned}
$$

for $t \geq t_{0}$ and $0 \leq u_{i}, v_{i} \leq \alpha, j=1,2, \cdots, k$, where $L(t) \in C_{r d}(\mathbb{T}, \mathbb{T})$;

(ii) $\int_{t_{0}}^{\infty} g_{m-1}(\sigma(s), 0) L(s) \Delta s<\infty$;

(iii) $\int_{t_{0}}^{\infty} g_{m-1}(\sigma(s), 0)|q(s)| \Delta s<\infty$;

(iv) there exists $p \in\left(\frac{1}{2}, 1\right)$ such that

$$
|p(t)| \leq 1-p, t \geq t_{0}
$$

(v) there exists $p \in(-1,0]$ such that

$$
p \leq p(t) \leq 0, t \geq t_{0}
$$

(vi) there exist $p_{1}, p_{2} \in(-\infty,-1)$ such that

$$
p_{1} \leq p(t) \leq p_{2}, t \geq t_{0}
$$

(vii) there exists $p \in(0,1)$ such that

$$
0<p(t) \leq p, t \geq t_{0}
$$

(viii) there exist $p_{1}, p_{2} \in(1,+\infty)$ such that

$$
p_{1} \leq p(t) \leq p_{2}, t \geq t_{0} .
$$

Theorem 2.1. Assume that (i), (ii), (iii) and (iv) hold, then Equation (1) has a bounded nonoscillatory solution which is bounded away from zero.

Proof. Choose $d_{1}, c_{1}$ such that $0<d_{1}<(2 p-1) \alpha$ and $d_{1}+(1-p) \alpha<c_{1}<p \alpha$. Let

$c=\min \left\{\frac{p}{2} \alpha, p \alpha-c_{1}, c_{1}-d_{1}-(1-p) \alpha\right\}$. There exists a $t_{1} \geq t_{0}$ large enough such that when $t \geq t_{1}$, we have $\tau(t), \tau_{i}(t) \geq t_{0}, i=1,2, \cdots, k$ and

$$
\int_{t_{1}}^{\infty} g_{m-1}(\sigma(s), 0)[\alpha L(s)+|q(s)|] \Delta s \leq c .
$$

By condition (i) and the hypotheses on $f\left(t, u_{1}, \cdots, u_{k}\right)$, for any $t \geq t_{0}, 0 \leq u_{i} \leq \alpha, i=1,2, \cdots, k$, we have

$$
f\left(t, u_{1}, \cdots, u_{\mathrm{k}}\right) \leq \alpha L(t) .
$$

We define a set $\Omega \subset B C$ as follows:

$$
\Omega=\left\{x \in B C: d_{1} \leq x(t) \leq \alpha, t \geq t_{0}\right\} .
$$

Then $\Omega$ is a closed, bounded and convex subset of $B C$. Define a map $\Gamma$ on $\Omega$ as follows:

$$
\begin{aligned}
& (\Gamma x)(t) \\
& =\left\{\begin{array}{l}
c_{1}-p(t) x(\tau(t))+(-1)^{m-1} \int_{t}^{\infty} g_{m-1}(\sigma(s), t) \\
\cdot\left[f\left(s, x\left(\tau_{1}(s)\right),\left(\tau_{2}(s)\right), \cdots,\left(\tau_{k}(s)\right)\right)-q(s)\right] \Delta s, \\
t \geq t_{1}, \\
(\Gamma x)\left(t_{1}\right), t_{0} \leq t \leq t_{1} .
\end{array}\right.
\end{aligned}
$$

First, we shall show that for any $x \in \Omega$ and $t \geq t_{0}$, $(\Gamma x)(t) \in \Omega$. For any $x \in \Omega$ and $t \geq t_{1}$, by (2), (3) and (4), we get

$(\Gamma x)(t)$

$$
\begin{aligned}
& \geq c_{1}-p(t) x(\tau(t))-\int_{t}^{\infty} g_{m-1}(\sigma(s), t) \\
& {\left[f\left(s, x\left(\tau_{1}(s)\right), x\left(\tau_{2}(s)\right), \cdots, x\left(\tau_{\mathrm{k}}(s)\right)\right)+|q(s)|\right] \Delta s} \\
& \geq c_{1}-|p(t)| x(\tau(t)) \\
& -\int_{t}^{\infty} g_{m-1}(\sigma(s), 0)[\alpha L(s)+|q(s)|] \Delta s \\
& \geq c_{1}-(1-p) \alpha-\left[c_{1}-d_{1}-(1-p) \alpha\right]=d_{1} .
\end{aligned}
$$

Furthermore, we have

$$
(\Gamma x)(t)
$$

$$
\leq c_{1}-|p(t)| x(\tau(t))-\int_{t}^{\infty} g_{m-1}(\sigma(s), t)
$$$$
\left[f\left(s, x\left(\tau_{1}(s)\right), x\left(\tau_{2}(s)\right), \cdots, x\left(\tau_{\mathrm{k}}(s)\right)\right)+|q(s)|\right] \Delta s
$$

$\leq c_{1}+(1-p) \alpha$

$$
+\int_{t}^{\infty} g_{m-1}(\sigma(s), 0)[\alpha L(s)+|q(s)|] \Delta s
$$

$\leq c_{1}+(1-p) \alpha+p \alpha-c_{1}=\alpha$. 
Hence when $t \geq t_{0}$, we obtain $d_{1} \leq(\Gamma x)(t) \leq \alpha$, so $(\Gamma x)(t) \in \Omega$ for any $x \in \Omega$.

Next, we show that $\Gamma$ is a contraction mapping on $\Omega$. In fact for any $x, y \in \Omega$ and $t \geq t_{1}$, we have

$(\Gamma x)(t)$

$$
\begin{aligned}
& \leq|p(t)||x(\tau(t))-y(\tau(t))| \\
& +\int_{t}^{\infty} g_{m-1}(\sigma(s), t) \mid f\left(s, x\left(\tau_{1}(s)\right), x\left(\tau_{2}(s)\right), \cdots, x\left(\tau_{\mathrm{k}}(s)\right)\right) \\
& -f\left(s, y\left(\tau_{1}(s)\right), y\left(\tau_{2}(s)\right), \cdots, y\left(\tau_{\mathrm{k}}(s)\right)\right) \mid \Delta s \\
& \leq(1-p)\|x-y\|+\int_{t}^{\infty} g_{m-1}(\sigma(s), 0) L(s)\|x-y\| \Delta s \\
& \leq\left(1-p+\frac{p}{2}\right)\|x-y\|=\left(1-\frac{p}{2}\right)\|x-y\| .
\end{aligned}
$$

Since $0<1-\frac{p}{2}<1$, we conclude that $\Gamma$ is a contraction mapping on $\Omega$. By the Banach fixed point theorem, $\Gamma$ has a fixed point $x^{*} \in \Omega$. By Lemma 2.1, it is easy to see that $x^{*}(t)$ is a bounded nonoscillatory solution of the Equation (1). This completes the proof of Theorem 2.1.

Theorem 2.2. Assume that (i), (ii), (iii) and (v) hold, then Equation (1) has a bounded nonoscillatory solution which is bounded away from zero.

Proof. Choose $\beta>0$, such that $\beta \leq \frac{2(1+p) \alpha}{3}$. Obviously $(1+p) \alpha-\beta \geq \frac{\beta}{2}$. There exists a $t_{1} \geq t_{0}$ sufficiently large such that when $t \geq t_{1}$, we have $\tau(t)$, $\tau_{i}(t) \geq t_{0}, i=1,2, \cdots, k$ and

$$
\int_{t_{1}}^{\infty} g_{m-1}(\sigma(s), 0)[\alpha L(s)+|q(s)|] \Delta s \leq \frac{\beta}{2} .
$$

We define a closed, bounded and convex subset $\Omega$ of $B C$ as follows:

$$
\Omega=\left\{x \in B C: \frac{\beta}{2} \leq x(t) \leq \alpha, t \geq t_{0}\right\} .
$$

Define a map $\Gamma$ on $\Omega$ as follows:

$$
(\Gamma x)(t)
$$

$$
=\left\{\begin{array}{l}
\beta-p(t) x(\tau(t))+(-1)^{m-1} \int_{t}^{\infty} g_{m-1}(\sigma(s), t) \\
\cdot\left[f\left(s, x\left(\tau_{1}(s)\right), x\left(\tau_{2}(s)\right), \cdots, x\left(\tau_{k}(s)\right)\right)-q(s)\right] \Delta s, \\
t \geq t_{1}, \\
(\Gamma x)\left(t_{1}\right), t_{0} \leq t \leq t_{1} .
\end{array}\right.
$$

The rest of the proof is similar to that of Theorem 2.1 and hence omitted. The proof is complete.

Theorem 2.3. Assume that (i), (ii), (iii) and (vi) hold. $\tau$ has the inverse $\tau^{-1} \in C(\mathbb{T}, \mathbb{T})$, then Equation (1) has a bounded nonoscillatory solution which is bounded away from zero.

Proof. We choose positive constants $M_{1}, M_{2}, \beta$, such that $M_{2} \leq \alpha,-p_{1} M_{1}<\beta<\left(-p_{2}-1\right) M_{2}$. Let

$$
c=\min \left\{\frac{\beta+p_{1} M_{1}}{p_{1}} p_{2},\left(-p_{2}-1\right) M_{2}-\beta,-\frac{1+p_{2}}{2} \alpha\right\} .
$$

There exists a $t_{1} \geq t_{0}$ large enough such that when $t \geq t_{1}$, we have $\tau^{-1}\left(\tau_{i}(t)\right) \geq t_{0}, i=1,2, \cdots, k$, and

$$
\int_{t^{-1}(t)}^{\infty} g_{m-1}(\sigma(s), 0)[\alpha L(s)+|q(s)|] \Delta s \leq c .
$$

We define a closed, bounded and convex subset $\Omega$ of $B C$ as follows:

$$
\Omega=\left\{x \in B C: M_{1} \leq x(t) \leq M_{2}, t \geq t_{0}\right\} .
$$

Define a map $\Gamma: \Omega \rightarrow B C$ as follows:

$(\Gamma x)(t)=$

$$
\left\{\begin{array}{l}
-\frac{\beta}{p\left(\tau^{-1}(t)\right)}-\frac{x\left(\tau^{-1}(t)\right)}{p\left(\tau^{-1}(t)\right)}+\frac{(-1)^{m-1}}{p\left(\tau^{-1}(t)\right)} \int_{t^{-1}(t)}^{\infty} g_{m-1}(\sigma(s), t) \\
\cdot\left[f\left(s, x\left(\tau_{1}(s)\right), x\left(\tau_{2}(s)\right), \cdots, x\left(\tau_{k}(s)\right)\right)-q(s)\right] \Delta s, \\
t \geq t_{1}, \\
(\Gamma x)(t), t_{0} \leq t \leq t_{1} .
\end{array}\right.
$$

First, we shall show that $\Gamma \Omega \subset \Omega$. For any $x \in \Omega$ and $t \geq t_{1}$, note that

$$
\begin{aligned}
&(\Gamma x)(t) \\
& \geq-\frac{\beta}{p\left(\tau^{-1}(t)\right)} \\
&+ \frac{1}{p\left(\tau^{-1}(t)\right)} \int_{t^{-1}(t)}^{\infty} g_{m-1}(\sigma(s), t)[\alpha L(s)+|q(s)|] \Delta s \\
& \geq-\frac{\beta}{p_{1}}+\frac{1}{p_{2}} \int_{\tau^{-1}(t)}^{\infty} g_{m-1}(\sigma(s), 0)[\alpha L(s)+|q(s)|] \Delta s \\
& \geq-\frac{\beta}{p_{1}}+\frac{\left(\beta+p_{1} M_{1}\right) p_{2}}{p_{1} p_{2}}=M_{1}
\end{aligned}
$$

and

$$
(\Gamma x)(t) \leq-\frac{\beta}{p_{2}}-\frac{M_{2}}{p_{2}}-\frac{\left(-p_{2}-1\right) M_{2}-\beta}{p_{2}}=M_{2} .
$$

Thus $(\Gamma x)(t) \in \Omega$ for $x \in \Omega$, this is $\Gamma \Omega \subset \Omega$.

Next, we show that $\Gamma$ is a contraction mapping on $\Omega$. In fact for any $x, y \in \Omega$ and $t \geq t_{1}$, we have 


$$
\begin{aligned}
& (\Gamma x)(t) \leq-\frac{1}{p\left(\tau^{-1}(t)\right)}\left|x\left(\tau^{-1}(t)\right)-y\left(\tau^{-1}(t)\right)\right| \\
& -\frac{1}{p\left(\tau^{-1}(t)\right)} \int_{\tau^{-1}(t)}^{\infty} g_{m-1}(\sigma(s), t) \mid f\left(s, x\left(\tau_{1}(s)\right), x\left(\tau_{2}(s)\right),\right. \\
& \left.\cdots, x\left(\tau_{k}(s)\right)\right)-f\left(s, y\left(\tau_{1}(s)\right), y\left(\tau_{2}(s)\right), \cdots, y\left(\tau_{k}(s)\right)\right) \mid \Delta s \\
& \leq-\frac{1}{p_{2}}\|x-y\|-\frac{1}{p_{2}} \int_{\tau^{-1}(t)}^{\infty} g_{m-1}(\sigma(s), 0) L(s)\|x-y\| \Delta s \\
& \leq \frac{1}{p_{2}}\left(-1+\frac{1+p_{2}}{2}\right)\|x-y\|=\frac{p_{2}-1}{2 p_{2}}\|x-y\| .
\end{aligned}
$$

Since $0<\frac{p_{2}-1}{2 p_{2}}<1$, we conclude that $\Gamma$ is a contraction mapping on $\Omega$. By the Banach fixed point theorem, $\Gamma$ has a fixed point $x^{*} \in \Omega$. By Lemma 2.1, it is easy to see that $x^{*}(t)$ is a bounded nonoscillatory solution of the Equation (1). This completes the proof of Theorem 2.3.

Theorem 2.4. Assume that (i), (ii), (iii) and (vii) hold, then equation (1) has a bounded nonoscillatory solution which is bounded away from zero.

Proof. Choose $\beta>0$, such that $p \alpha<\beta<\alpha$. Let $c=\min \left\{\alpha-\beta, \frac{\beta-p \alpha}{2}\right\}$. There exists a $t_{1} \geq t_{0}$ large enough such that when $t \geq t_{1}$, we have

$$
\tau(t), \tau_{i}(t) \geq t_{0}, i=1,2, \cdots, k,
$$

and

$$
\int_{t_{1}}^{\infty} g_{m-1}(\sigma(s), 0)[\alpha L(s)+|q(s)|] \Delta s \leq c .
$$

Easily to know

$$
\Omega=\left\{x \in B C: \frac{\beta-p \alpha}{2} \leq x(t) \leq \alpha, t \geq t_{0}\right\}
$$

is a closed, bounded and convex subset of $B C$. Define a map $\Gamma: \Omega \rightarrow B C$ as follows:

$$
\begin{aligned}
& (\Gamma x)(t) \\
& =\left\{\begin{array}{l}
\beta-p(t) x(\tau(t))+(-1)^{m-1} \int_{t}^{\infty} g_{m-1}(\sigma(s), t) \\
\cdot\left[f\left(s, x\left(\tau_{1}(s)\right), x\left(\tau_{2}(s)\right), \cdots, x\left(\tau_{k}(s)\right)\right)-q(s)\right] \Delta s, \\
t \geq t_{1}, \\
(\Gamma x)\left(t_{1}\right), t_{0} \leq t \leq t_{1} .
\end{array}\right.
\end{aligned}
$$

The rest of the proof is similar to that of Theorem 2.1 and hence omitted. The proof is complete.

Theorem 2.5. Assume that $\alpha \geq 1$, (i), (ii), (iii) and (viii) hold. $\tau$ has the inverse $\tau^{-1} \in C(\mathbb{T}, \mathbb{T})$, then Equation (1) has a bounded nonoscillatory solution which is bounded away from zero.

Proof. We choose $\beta$, such that $1<\beta<p_{1}$. Let $c=\min \left\{\beta-1, \frac{p_{1}-\beta}{2}, \frac{\beta-1}{2} \alpha\right\}$. There exists a $t_{1} \geq t_{0}$ large enough such that when $t \geq t_{1}$, we have $\tau^{-1}\left(\tau_{i}(t)\right) \geq t_{0}, i=1,2, \cdots, k$, and

$$
\int_{\tau^{-1}(t)}^{\infty} g_{m-1}(\sigma(s), 0)[\alpha L(s)+|q(s)|] \Delta s \leq c .
$$

We define a closed, bounded and convex subset $\Omega$ of $B C$ as follows:

$$
\Omega=\left\{x \in B C: \frac{p_{1}-\beta}{2 p_{2}} \leq x(t) \leq \frac{p_{1}+\beta}{2 p_{1}}, t \geq t_{0}\right\} .
$$

Define a map $\Gamma: \Omega \rightarrow B C$ as follows:

$$
\begin{aligned}
& (\Gamma x)(t)= \\
& \left\{\begin{array}{l}
\frac{\beta}{p\left(\tau^{-1}(t)\right)}-\frac{x\left(\tau^{-1}(t)\right)}{p\left(\tau^{-1}(t)\right)}+\frac{(-1)^{m-1}}{p\left(\tau^{-1}(t)\right)} \int_{\tau^{-1}(t)}^{\infty} g_{m-1}(\sigma(s), t) \\
\cdot\left[f\left(s, x\left(\tau_{1}(s)\right), x\left(\tau_{2}(s)\right), \cdots, x\left(\tau_{\mathrm{k}}(s)\right)\right)-q(s)\right] \Delta s, \\
t \geq t_{1}, \\
(\Gamma x)\left(t_{1}\right), t_{0} \leq t \leq t_{1} .
\end{array}\right.
\end{aligned}
$$

The rest of the proof is similar to that of Theorem 2.3 and hence omitted. The proof is complete.

Remark 2.1. Theorem 1 - 5 not only unify the known results for differential and difference equations corresponding to Equation (1), but also generalize and improve essentially the existing results of [13-15] using the time scale theory.

We will give the following examples to illustrate our mainresults.

Example 2.1. Consider the forth-order dynamic equation on the time scale $\mathbb{T}=\left\{q^{n}: n \in \mathbb{N}_{0}, q>1\right\}$

$$
\begin{aligned}
& \left(x(t)-\frac{1}{\sqrt{q}} x\left(\frac{t}{q}\right)\right)^{\Delta^{4}}+\frac{(1-\sqrt{q})(q+1)^{2}\left(q^{2}+1\right)}{q^{10}} \\
& \times \frac{\left(q^{2}+q+1\right)}{t^{2}\left(t+q^{3}\right)^{3}} x^{3}\left(\frac{t}{q^{3}}\right) \\
& =2 \frac{(1-\sqrt{q})(q+1)^{2}\left(q^{2}+1\right)\left(q^{2}+q+1\right)}{q^{10} t^{5}}
\end{aligned}
$$

Here $m=4, p(t)=-\frac{1}{\sqrt{q}}, \tau(t)=\frac{t}{q}$,

$$
\begin{aligned}
& q(t)=2 \frac{(1-\sqrt{q})(q+1)^{2}\left(q^{2}+1\right)\left(q^{2}+q+1\right)}{q^{10} t^{5}}, \\
& L(t)=3 \alpha^{2} \frac{\left|(1-\sqrt{q})(q+1)^{2}\left(q^{2}+1\right)\left(q^{2}+q+1\right)\right|}{q^{10} t^{2}\left(t+q^{3}\right)^{3}} .
\end{aligned}
$$


By the definition of $g_{k}(s, t)$, we have

$$
\begin{aligned}
& g_{4-1}(\sigma(s), 0) L(s) \\
& \leq s^{3} \frac{3 \alpha^{2}(\sqrt{q}-1)(q+1)^{2}\left(q^{2}+1\right)}{q^{10}} \frac{\left(q^{2}+q+1\right)}{s^{2}\left(s+q^{3}\right)^{3}} \\
& \leq \frac{3 \alpha^{2}(\sqrt{q}-1)(q+1)^{2}\left(q^{2}+1\right)\left(q^{2}+q+1\right)}{q^{10} s^{2}}, \\
& g_{4-1}(\sigma(s), 0)|q(s)| \\
& \leq s^{3} 2 \frac{(\sqrt{q}-1)(q+1)^{2}\left(q^{2}+1\right)\left(q^{2}+q+1\right)}{q^{10} s^{5}} \\
& =2 \frac{(\sqrt{q}-1)(q+1)^{2}\left(q^{2}+1\right)\left(q^{2}+q+1\right)}{q^{10} s^{2}} .
\end{aligned}
$$

Then

$$
\begin{gathered}
\int_{t_{0}}^{\infty} \frac{3 \alpha^{2}(\sqrt{q}-1)(q+1)^{2}\left(q^{2}+1\right)\left(q^{2}+q+1\right)}{q^{10} s^{2}} \Delta s<\infty, \\
\int_{t_{0}}^{\infty} 2 \frac{(\sqrt{q}-1)(q+1)^{2}\left(q^{2}+1\right)\left(q^{2}+q+1\right)}{q^{10} s^{2}} \Delta s<\infty .
\end{gathered}
$$

It is obvious that Equation (5) satisfies all conditions of Theorem 2.2. Hence Equation (5) has a bounded nonoscillatory solution which is bounded away from zero. In fact $x(t)=1+\frac{1}{t}$ is a solution of Equation (5). However, to the best of our knowledge, there are no results dealing with the existence of nonoscillatory solutions for Equation (5).

Example 2.2. Consider the third-order dynamic equation on the time scale $\mathbb{T}=\mathbb{N}$

$$
(x(t)-2 x(t-1))^{\Delta^{3}}+\frac{1}{2^{t}} x(t-1)=\frac{11\left(2^{t}\right)+16}{8\left(2^{2 t}\right)},
$$

$t \geq 2$.

Here $m=3, p(t)=-2, \tau(t)=t-1$,

$$
f\left(t, x\left(\tau_{1}(t)\right)\right)=\frac{1}{2^{t}} x(t-1)
$$

and $q(t)=\frac{11\left(2^{t}\right)+16}{8\left(2^{2 t}\right)}$. It is easy to see that all conditions of Theorem 2.3 are satisfied and hence Equation (6) has a bounded nonoscillatory solution which is bounded away from zero. In fact $x(t)=1+\frac{1}{2^{t}}$ is a solution of Equation (6).

\section{Acknowledgements}

The authors sincerely thank the reviewers for their valuable suggestions and useful comments that have lead to the present improved version of the original manuscript.

\section{REFERENCES}

[1] S. Hilger, "Analysis on Measure Chains-A Unified Approach to Continuous and Discrete Calculus," Results in Mathematics, Vol. 18, No. 1-2, 1990, pp. 18-56.

[2] R. Agarwal, M. Bohner, D. O'Regan and A. Peterson, "Dynamic Equations on Time Scales: A Survey," Journal of Computational and Applied Mathematics, Vol. 141, No. 1-2, 2002, pp. 1-26. doi:10.1016/S0377-0427(01)00432-0

[3] M. Bohner and A. Peterson, "Dynamic Equations on Time Scales: An Introduction with Applications," Birkhäuser, Boston, 2001.

[4] M. Bohner and A. Peterson, "Advances in Dynamic Equations on Time Scales," Birkhäuser, Boston, 2003. doi:10.1007/978-0-8176-8230-9

[5] B. G. Zhang and S. L. Zhu, "Oscillation of Second-Order Nonlinear Delay Dynamic Equations on Time Scales," Computers \& Mathematics with Applications, Vol. 49, No. 4, 2005, pp. 599-609.

[6] Q. L. Li and Z. Zhang, "Existence of Solutions to Nth Order Neutral Dynamic Equations on Time Scale," Electronic Journal of Differential Equations, Vol. 2010, No. 151, 2010, pp. 1-8. http://ejde.math.txstate.edu or http://ejde.math.unt.edu, ftp ejde.math.txstate.edu

[7] D. X. Chen, "Oscillation and Asymptotic Behavior for Nth-Order Nonlinear Neutral Delay Dynamic Eqautions on Time Scales," Acta Applicandae Mathematicae, Vol. 109, No. 3, 2010, pp. 703-719. doi:10.1007/s10440-008-9341-0

[8] T. S. Hassan, "Oscillation of Third Order Nonlinear Delay Dynamic Equations on Time Scales," Mathematical and Computer Modelling, Vol. 49, No. 7-8, 2009, pp. 15731586. doi:10.1016/j.mcm.2008.12.011

[9] Z. Q. Zhu and Q. R. Wang, "Existence of Nonoscillatory Solutions to Neutral Dynamic Equations on Time Scales," Journal of Mathematical Analysis and Applications, Vol. 335, No. 2, 2007, pp. 751-762. doi:10.1016/j.jmaa.2007.02.008

[10] T. Li, Z. Han, S. Sun and D. Yang, "Existence of Nonoscillatory Solutions to Second-Order Neutral Delay Dynamic Equations on Time Scales," Advances in Difference Equations, Vol. 2009, 2009, pp. 1-10. doi: $10.1155 / 2009 / 562329$

[11] T. X. Sun, H. Xi, X. Peng and W. Yu, "Nonoscillatory Solutions for Higher-Order Neutral Dynamic Equations on Time Scales," Abstract and Applied Analysis, Vol. 2010, 2010, pp. 1-16. doi:10.1155/2010/428963

[12] B. G. Zhang and X. H. Deng, "Oscillation of Delay Differential Equations on Time Scales," Mathematical and Computer Modelling, Vol. 36, No. 11-13, 2002, pp. 13071318. doi:10.1016/S0895-7177(02)00278-9

[13] B. G. Zhang and Y. J. Sun, "Existence of Nonoscillatory 
Solutions of a Class of Nonlinear Difference Equations with a Forced Term," Mathematica Bohemica, Vol. 126, No. 3, 2001, pp. 639-647.

[14] Y. Zhou and B. G. Zhang, "Existence of Nonoscillatory Solutions of Higher-Order Neutral Delay Difference Equations," Computers \& Mathematics with Applications,
Vol. 45, No. 6-9, 2003, pp. 991-1000. doi:10.1016/S0898-1221(03)00074-9

[15] W. D. Lu, "Existence of Nonoscillatory Solutions of First Order Nonlinear Neutral Equations," Journal of the Australian Mathematical Society Series B, Vol. 32, No. 2, 1990, pp. 180-192. 